\title{
PAX3: A Molecule with Oncogenic or Tumor Suppressor Function Is Involved in Cancer
}

\author{
Ashok Arasu, ${ }^{1}$ Sengottuvelan Murugan, ${ }^{2}$ Musthafa Mohamed Essa $\mathbb{D}^{,}{ }^{3}$ \\ Thirunavukkarasu Velusamy $\left(\mathbb{D},{ }^{1}\right.$ and Gilles J. Guillemin $\mathbb{1}^{4}{ }^{4}$ \\ ${ }^{1}$ Department of Biotechnology, School of Biotechnology and Genetic Engineering, Bharathiar University, Coimbatore 641046, \\ Tamil Nadu, India \\ ${ }^{2}$ Endocrine Research Facility, Department of Animal Science, Rutgers University, New Brunswick-08901, NJ, USA \\ ${ }^{3}$ Department of Food Science and Nutrition, CAMS, Sultan Qaboos University, Muscat, Oman \\ ${ }^{4}$ Neuroinflammation Group, Faculty of Medicine and Health Sciences, Deb Bailey MND Research Laboratory, \\ Macquarie University, NSW, 2109, Australia
}

Correspondence should be addressed to Musthafa Mohamed Essa; drmdessa@gmail.com, Thirunavukkarasu Velusamy; bharathiarasu@gmail.com, and Gilles J. Guillemin; gilles.guillemin@mq.edu.au

Received 8 February 2018; Accepted 2 July 2018; Published 16 August 2018

Academic Editor: Franco M. Buonaguro

Copyright (c) 2018 Ashok Arasu et al. This is an open access article distributed under the Creative Commons Attribution License, which permits unrestricted use, distribution, and reproduction in any medium, provided the original work is properly cited.

\begin{abstract}
Metastasis is the most deadly aspect of cancer and results from acquired gene regulation abnormalities in tumor cells. Transcriptional regulation is an essential component of controlling of gene function and its failure could contribute to tumor progression and metastasis. During cancer progression, deregulation of oncogenic or tumor suppressive transcription factors, as well as master cell fate regulators, collectively influences multiple steps of the metastasis cascade, including local invasion and dissemination of the tumor to distant organs. Transcription factor PAX3/Pax3, which contributes to diverse cell lineages during embryonic development, plays a major role in tumorigenesis. Mutations in this gene can cause neurodevelopmental disease and the existing literature supports that there is a potential link between aberrant expression of PAX3 genes in adult tissues and a wide variety of cancers. PAX3 function is tissue-specific and could contribute to tumorigenesis either directly as oncogene or as a tumor suppressor by losing its function. In this review, we discuss comprehensively the differential role played by PAX3 in various tissues and how its aberrant expression is implicated in disease development. This review particularly highlights the oncogenic and tumor suppressor role played by PAX3 in different cancers and underlines the importance of precisely identifying tissue-specific role of PAX3 in order to determine its exact role in development of cancer.
\end{abstract}

\section{Introduction}

Cancer is a disease that is characterized by uncontrolled cell growth, proliferation, migration, and invasion of abnormal cells resulting in aggregation of these cells to form tumors in organ. There are several etiologies for the development of cancer, ranging from environmental to genetic causes. Mutations that inhibit the normal function of oncogenes, tumor suppressor genes, and apoptosis genes can lead to uncontrollable cell growth. Changes in molecular mechanisms that regulate stem cell differentiation were also known to be a strong factor associated with development of cancer [1]. The paired box (PAX: human)/(Pax: mouse) gene family is known to be associated with developmental functions and play a crucial role in cellular proliferation, differentiation, migration, and tissue development [2]. PAX proteins are subclassified according to the additional DNAbinding homeodomain or octapeptide region, which can serve as a binding motif for the protein cofactor for effective inhibition of downstream gene transcriptions [3]. The role of PAX/Pax protein as transcriptional activators or repressors has been demonstrated through their interaction with other transcription factors to induce target promoters [4]. There is a possible link between the anomalous expression of PAX genes in adult tissues and a wide class of cancers by promoting tumorigenesis as an oncogene or by failing to perform its role 
as a tumor suppressor $[5,6]$. PAX proteins may be useful as a biomarker in the diagnosis of cancers. PAX genes have an oncogenic ability when constitutively expressed, either as a part of a fusion gene or as a whole gene [7-12].

PAX3, a member of PAX gene family, plays a critical role in cell proliferation, differentiation, and migration during embryonic development of cells [13]. PAX3 contains all three complete structural domains: a paired domain, homeodomain, and octopeptide, which is a characteristic of PAX family of proteins. PAX3 interacts with other transcription factors through its paired domain (PD) and recruits them to target promoters $[14,15]$. PAX3 is expressed as several isoforms such as PAX3a, PAX3b, PAX3c, PAX3d, PAX3e, PAX3g, and PAX3h [16-19]. Each of these isoforms are generally involved in developmental function, however, with specific functions that are unique to them in different tissues [19]. PAX3 expression is necessary for proliferation and migration of neural crest (NC) cells and muscle cell precursors in the dorsal dermomyotome. It is also involved in developmental pathways that lead to melanocytes and neurons originating from the NC and mature skeletal myocytes from the dorsal dermomyotome. Acceleration of proliferation, migration, and survival of neural crest cells are also regulated by PAX3 [20]. PAX3/Pax3 (human/mouse) is a developmentally expressed transcription factor in embryo and is rapidly switched off during terminal differentiation [21-23]. PAX3 is necessary not only for the proper specification of these developing tissues, but also for cell survival. Homozygous loss of PAX3 in mouse leads to several neural crest and neural tube defects, attributable to induction of p53-dependent apoptosis [5, 24-28]. PAX3 is necessary for neural crestderived peripheral nervous system development (PNS) and its reexpression in differentiated neural crest cells is suspected to be involved in the development of peripheral nervous system tumor, neuroblastoma. A gain of function mutation in PAX3 is associated with several diseases such as Waardenburg syndromes, craniofacial-deafness-hand syndromes, alveolar rhabdomyosarcomas, and neuroblastomas [3, 29]. Being an important developmental factor, the expression of PAX3 gradually decreases as the tissues differentiate. However, its reexpression in differentiated adult tissues that require PAX3 function during embryonic development leads to several tumors $[3,15]$.

More recently, PAX3 was observed to be overexpressed in various types of cancer tissues and cell lines like neuroblastomas, glioblastomas, melanomas, and gastric cancers, suggesting that it may function as an oncogene in these cancers [30-36]. In rhabdomyosarcomas, PAX3-FOXO1 fusion increases the transcriptional activity of PAX3 by constitutive nuclear localization [37]. However, in thyroid cancer, it plays a completely different role as a tumor suppressor. Loss of function of PAX3 due to decreased expression caused by hypermethylation is reasoned out as an underlying cause for development of thyroid cancer [36]. Based on these facts, it is important to determine the functions of PAX3 specific to tissue type and then understand its role in disease pathogenesis. This review will mainly discuss about the specific roles played by PAX3 in different tissue types and then will focus on specific examples where PAX3 has contradictory function as either oncogene or tumor suppressor, and is implicated in development of cancer.

\section{PAX3 and Its Role in Development and Diseases}

PAX3 is a very well-characterized protein that is expressed in the neural crest (NC) [38]. It is also one of the most wellcharacterized transcription factors of the NC and has been extensively studied in NC induction during development, cardiac NC, and melanocyte lineages [38]. The physiological function of PAX3 has been well understood through characterizing the mutations that are reported in mice and humans. PAX3 plays a major role during early skeletal muscle formation in the embryo, while PAX7 predominates during postnatal growth and muscle regeneration in the adult [39]. PAX3 is expressed during early migration of $\mathrm{NC}$ and in somitic cells along the neuronal crest cell migratory path [40]. PAX3 is generally extinguished in migratory neural crest cells as they differentiate but maintained in the melanocyte lineage and also sustained in cultured neural crest-derived stem cells [41], in later stages of development in various lineages. The timing of expression in early neural crest and subsequent downregulation as neural crest-derived cells differentiate also implicates PAX3 as an important factor for maintaining progenitor populations. In fact, persistent PAX3 missexpression in cranial neural crest cells resulted in cleft palate and other craniofacial defects, ocular defects, and perinatal lethality [42]. Another phenotype associated with persistent PAX3 expression is that bone morphogenetic protein- (BMP) induced osteogenesis is blocked via upregulation of the PAX3 target Sostdc1, a soluble BMP inhibitor. This highlights the role PAX3 plays in maintaining an undifferentiated state by blocking responsiveness to differentiation signals [42]. PAX3 function well-characterized in the neural crest is the availability of a series of mutations in the murine PAX3 gene. These alleles represent a range of null, severely hypomorphic, and mildly hypomorphic forms of PAX3. In addition to neural crest defects, PAX3 and PAX7 mutants display neurulation defects and altered somitogenesis [39]. PAX3 and versican have mutually exclusive expression pattern and when PAX3 is lost, the versican (VCAN) expression domain is expanded, correlating with an absence of migrating neural crest cells lateral to the neural tube [43]. Where PAX3 and Zicl expression overlap, they act together to specify the neuroectoderm to adopt a neural crest fate [44, 45] and activate Slug expression in a Wnt-dependent manner [24]. Multiple experiments demonstrate that PAX3 and Zic1 together are both necessary and sufficient to specify neural crest $[44,45]$; PAX3 is upregulated in the dorsal neural crest in response to Wnt signaling and also depends on bone morphogenetic protein (BMP) signaling. Downregulation of PAX3 occurs when the neural crest migration starts [46, 47].

In the absence of functional PAX3 (PAX3 mutants), the neural crest cells migrating to the peripheral nervous system undergo premature neurogenesis (evidenced by increased Brn3 positive staining in neural tube explants), perhaps due to a change in the regulation of genes such as Hesl and 
Ngn2 (needed for differentiation and proliferation), which PAX3 regulates by directly binding to their promoters. In this role, PAX3 may couple migration with neural crest cells maintenance and neurogenesis [48]. In vitro, a different phenomenon is seen. PAX3 is initially expressed in all neural crest cells from culture but is subsequently only retained in neurons. PAX3 homozygous mutant neural crest cultures had an $80 \%$ reduction in the capacity to generate sensorylike neurons. Downregulation of PAX3 in dorsal root ganglia inhibited $80-90 \%$ of newly generated sensory neurons but had no effect on survival of sensory neurons or precursors [49]. Mutation of PAX3, as occurs in PAX3 homozygotes, results in development of heart defects including persistent truncus arteriosus (PTA), signs of cardiac failure $[25,50$, 51], and defects of the aortic arches, in addition to thymus, thyroid, and parathyroid defects, ultimately leading to embryonic lethality. PAX3 inhibits p53-dependent apoptosis in the dorsal neural tube to regulate its closure [28] and is also important for normal development and migration of the cardiac NC to the developing heart [25]. In addition to its function as a standalone transcription factor, several downstream molecules of other important pathways can act as a coactivator in mediating the transcriptional effects of PAX3. The downstream effector molecules of the Hippo pathway Taz and Yap65 play an important role in neural crest development and deletion of these proteins in neural crest resulted in embryo-lethal neural crest defects and decreased expression of the Pax3 target gene, Mitf [52].

The role of PAX3 as a transcription factor is well established in the development of melanocytes during embryogenesis and has recently been characterized as a molecular switch in the initiation of mature melanocytes formation [32]. Interestingly, PAX3 is one of the key molecules in melanogenesis that is absent or greatly reduced in the bulbs of white hair when compared to black hair, further reinforcing its role in melanocyte stem cell populations [53]. Among various PAX3 isoforms, PAX3c is the most studied and wellcharacterized isoform; in mouse melanocytes in vitro, the effect of PAX3 on proliferation, migration, survival, and transformation varies depending on the isoform, with some isoforms having opposite effects [20].

Evidence supports a role for PAX3 as a controller of a cascade of transcriptional events that are necessary and sufficient for skeletal myogenesis $[54,55]$. PAX3 is downregulated when muscle tissue begins to differentiate and the muscle specific transcription factors are activated $[54,56]$. Ectopic expression of PAX3 prevents the myogenic differentiation of myoblasts into myotubes, which might involve the cooperation of $M s \times 1$ and Notch genes $[57,58]$.

Aberrant expression of PAX proteins is implicated in developmental defects. Specific mutations within a number of PAX/ Pax (human/mouse) genes lead to a range of developmental abnormalities in both human beings and mouse. Many studies proved that PAX3 loss of function mutations are involved in either type 1 or type 3 Waardenburg syndrome [59-65]. PAX3/Pax3 (human/mouse) mutations are associated with limb muscle hypoplasia in Waardenburg syndrome patients and Splotch phenotype mice, respectively $[54,55]$.
Interestingly, PAX3 mutations were typically not found in families with other neurocristopathies [64]. Mutations that partially abolish the activity of PAX2, PAX3, and PAX6 were known to cause dysmorphic syndromes with developmental abnormalities [66-70]. PAX2 and PAX8 double mutants show a complete lack of kidney formation due to reduced activity of these proteins [71].

\section{PAX3 Plays an Oncogenic Role in Various Types of Tumors}

Several members of the PAX family, especially subgroups II (PAX2, PAX5, and PAX8 ) and III (PAX3 and PAX7), play key roles in human malignancies, such as renal tumors, lymphoma, medullary thyroid carcinoma, rhabdomyosarcoma (RMS), and melanoma [5, 72-74]. Transfection of 3T3 cells with wild-type Pax1, Pax2, Pax3, Pax6, or Pax8 produced tumors in the nude mice within 2 to 6 weeks; the tumors were well vascularized and resembled spindle cell sarcomas, with high and a typical mitotic activity and infiltration into nerve and muscle tissues and blood vessels [75]. PAX genes in subgroups I (PAX1 and PAX9) are less often involved in cancer or their expression is indicative of a more favorable outcome [5]. PAX/Pax (human/mouse) proteins, therefore, serve as tumor markers in several cancers such as rhabdomyosarcoma, melanoma, neuroblastoma, and Ewing's sarcoma [2, 6, 76]. While PAX expression is very rare in adult tissues, findings support that its expression may be involved in maintaining pluripotency and survival of stem cell populations. Either continuing or recurring PAX expression is essential to provide pools of progenitor cells for tissue regeneration upon injury. In cancer cells, achieving self-sufficiency in growth signals and unrestricted replicative potential are required to able to survive in potentially adverse microenvironments during tumor progression. There are numerous studies that prove that PAX genes play important roles in conferring growth and survival advantages to cancer cells and that they regulate cell plasticity [2]. Conceptually, cell proliferation and differentiation are placed at opposite ends of the "spectrum" of tumor progression. PAX genes, such as PAX8, could play a key role in balancing these processes [2].

PAX3, which plays a critical role during neural crest development, especially in progenitor cell populations, also plays a role in tumor formation in these tissue types. Many human neuroectodermal tumors express PAX3 $[18,34,77,78]$. The role of PAX3 and its isoforms in myogenesis, melanogenesis, neurogenesis, and related oncogenesis have been well established (Figure 1). PAX3 is specifically detected in most neural crest-derived Ewing's family tumors and in most peripheral neuroectodermal tumors [34], small cell lung cancer [18], and most primary cultured melanomas $[18,34,79]$. Screening of Ewing's sarcoma and peripheral neuroectodermal tumor specimens and its derived cell lines showed elevated PAX3 expression [34]. Deactivation of p53 during neural tube closure and cardiac neural crest development by stimulating its ubiquitination and degradation requires $\mathrm{PAX} 3$ function, but not as a transcriptional regulator 


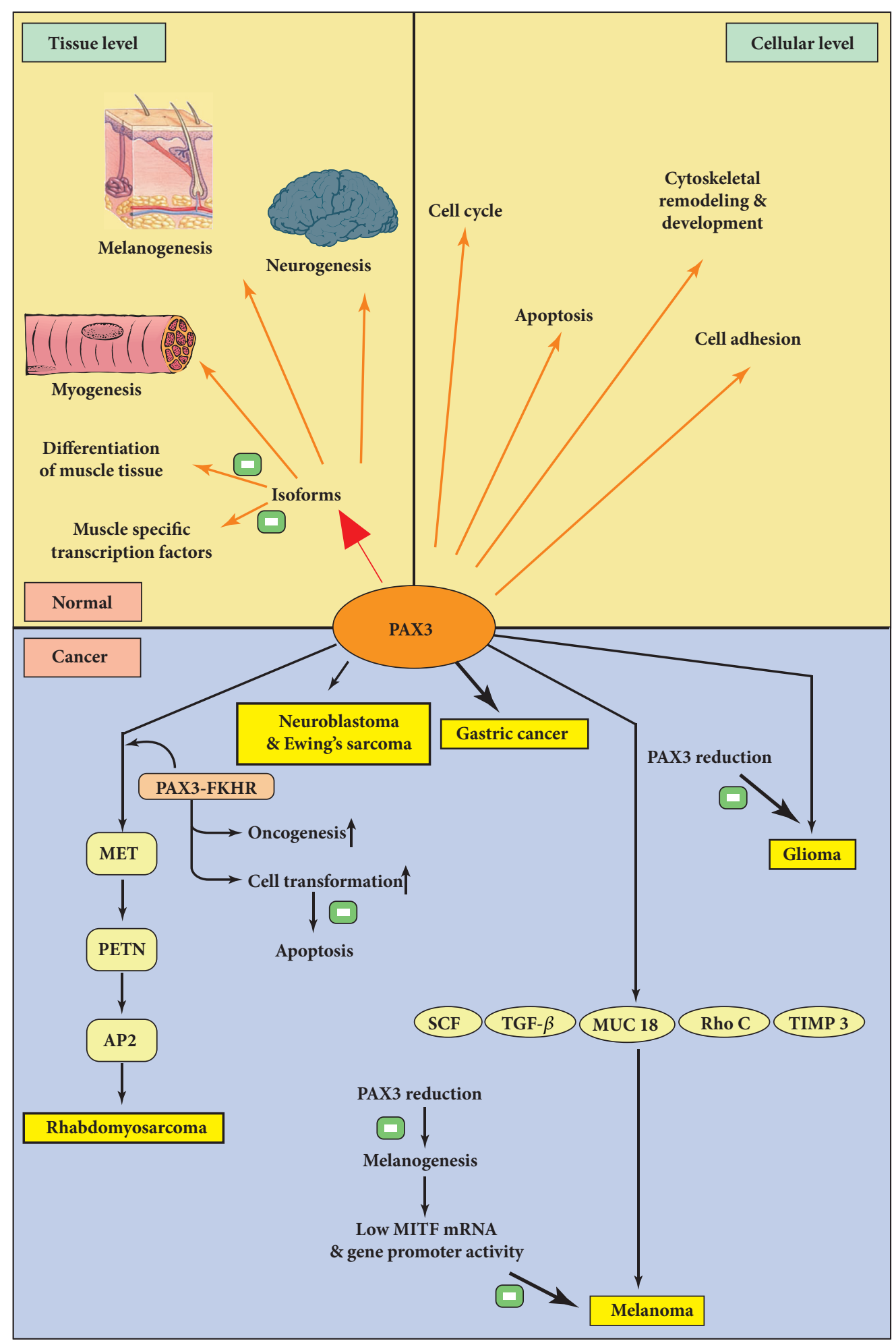

FIGURE 1: Oncogenic role of PAX3 in various types of cancer. PAX3 isoforms play a role in myogenesis, melanogenesis, and neurogenesis. PAX3 is involved in physiological events such as cell cycle, apoptosis, cell adhesion, and cytoskeletal remodelling and development. Overexpression of PAX3 inhibits the differentiation of muscle tissues and muscle specific transcription factors. While overexpression of PAX3 was observed in various types of cancers, PAX3-FKHR leads to tumorigenesis in rhabdomyosarcoma through altering the MET, PTEN, or AP2 signaling pathways. PAX3-FKHR can induce cellular transformation and prevent apoptosis. Reduction of PAX3 prevents development of glioma and also induces a loss of melanogenesis in melanoma. MITF mRNA and gene promoter activity is also decreased because of the PAX3 reduction. Melanoma susceptibility and progression genes like SCF, TGF- $\beta$, MUC18, RhoC, and TIMP3 can be regulated by PAX3 and its leads to melanoma. 
TABLE 1: Pax3 transcription factor plays a dual role in various types of cancers.

\begin{tabular}{|c|c|c|}
\hline Cancer Type & Effects & Role of PAX3 \\
\hline Rhabdomyosarcoma & \multirow{6}{*}{ Oncogenic effects } & $\begin{array}{l}\text { PAX3-FKHR can induce cellular transformation and prevent apoptosis by altering } \\
\text { the MET, PTEN or AP2 signalling pathways }[55,57,58,87,89,90,92] \text {. }\end{array}$ \\
\hline Melanoma & & $\begin{array}{l}\text { Regulating the expression of a variety of melanocytic genes, followed by a decrease } \\
\text { in MITF mRNA and gene promoter activity and progression genes SCF, TGF- } \beta \text {, } \\
\text { MUC18, RhoC and TIMP }[84,85]\end{array}$ \\
\hline Neuroblastoma & & $\begin{array}{l}\text { Inhibition of PAX3 expression significantly decreased the attachment of S-type } \\
\text { SH-EP1 cells to extra-cellular matrix proteins, fibronectin, laminin and collagen IV } \\
\text { and therefore downregulation of PAX3 reduces migration and invasion [31]. }\end{array}$ \\
\hline Glioblastoma & & $\begin{array}{c}\text { PAX3 was upregulated in the glioma SHG-44 cells promoted tumor formation in } \\
\text { vivo cell lines, while its knockdown resulted in decreased cell proliferation, invasion } \\
\text { and induced apoptosis in these cells. PAX3 expression in glioblastoma U-87MG } \\
\text { cells suppressed tumorigenicity [30]. }\end{array}$ \\
\hline Gastric cancer & & $\begin{array}{l}\text { MiR-206 significantly suppressed gastric cancer cell invasion and metastasis both in } \\
\text { vitro and in vivo by down regulating PAX3. In vivo metastasis assay shows that } \\
\text { overexpression of PAX3 promotes invasiveness and pulmonary metastasis [35]. }\end{array}$ \\
\hline Urothelial cancer & & $\begin{array}{c}\text { FOXO3a negatively regulates Twistl and Y-box-binding protein } 1 \text { (YB-1), and } \\
\text { positively regulated E-cadherin in With FOXO3a PAX3 acts as a prognostic factor } \\
\text { [94]. }\end{array}$ \\
\hline Thyroid cancer & \multirow[t]{3}{*}{ Suppressor } & $\begin{array}{l}\text { PAX3 a tumor suppressor in thyroid cancer, through inhibiting the activities of } \\
\text { PI3K/Akt and MAPK signaling pathways and promoting transcription factor } \\
\text { FOXO3a.). The ectopic expression of PAX3, up-regulated the expression of ZIC1, a } \\
\text { zinc-finger transcription factor, which in turn increases FOXO3a transcriptional } \\
\text { activity [36]. }\end{array}$ \\
\hline Rhabdomyosarcoma & & $\begin{array}{c}\text { Overexpression of miR-29 and } 206 \text { downregulates the expression of cell cycle genes, } \\
\text { induce cell cycle arrest through stabilizing PAX3 [6]. }\end{array}$ \\
\hline Alveolar rhabdomyosarcoma & & PAX3-FOXO1 up-regulate Noxa (Promote activation of caspases and apoptosis) [95]. \\
\hline
\end{tabular}

indicating a novel role for PAX3 in human diseases, such as in neural crest-derived cancers and Waardenburg syndrome types 1 and 3 [80].

Neuroblastoma is an extracranial solid tumor that occurs in children. Abnormally elevated levels of PAX3 expression has also been found in some neuroblastoma cell lines and tumors [81]. PAX-3 is overexpressed in glioma tissues compared to normal brain tissues; however, the pathogenic role of PAX-3 in human glioma cells remains to be elucidated. PAX3 was upregulated in the glioma cell lines, while its knockdown resulted in decreased cell proliferation and invasion and induced apoptosis in these cells (Figure 1). Inhibition of PAX3 expression in glioblastoma U-87MG cells suppressed tumorigenicity and upregulation of PAX3 expression in glioma SHG-44 cells promoted tumor formation in vivo. These results indicate that PAX3 in glioma is essential for gliomagenesis; thus, targeting PAX3 or its downstream targets may lead to novel therapies for this disease [30]; this data has been represented in Table 1 . PAX3 contributes to various cell lineages during embryonic development and is also important in tumourigenesis. Reexpression of PAX3 was found in neuroblastoma cells, and the malignant neuroblastic (N-type) neuroblastoma cells expressed significantly higher PAX3 protein expression in comparison to their benign substrate-adherent (S-type) counterparts. PAX3 knockdown resulted in persistent cell growth inhibition in neuroblastoma cell, through G1 cell cycle arrest, and leads to progressive apoptosis.
Inhibition of PAX3 expression significantly decreased the attachment of S-type SH-EP1 cells to extracellular matrix proteins, fibronectin, laminin, and collagen IV. Migration and invasion of both neuroblastoma cell types were markedly reduced after PAX3 downregulation. Microarray analyses revealed that signaling pathways involving cell cycle, apoptosis, cell adhesion, cytoskeletal remodelling, and development were altered in particular, by PAX3 downregulation (Table 1). These novel findings emphasize that PAX3 might contribute to oncogenic characteristics of neuroblastoma cells by regulating a variety of crucial signaling pathways [31].

While PAX3 promotes melanocyte phenotype, it plays an indispensable role in stem cell maintenance and actively promotes lesion survival, while BRAF mutations support tumor proliferation in melanoma [32]. PAX3 also plays a main role, in the pathogenesis of melanoma by influencing pathways through its transcriptional activity [82] and also by inducing p53 loss of function by promoting its ubiquitination [80]. $\mathrm{PAX} 3$ is a key transcription factor in regulating the expression of a variety of melanocytic genes [22, 83]. Inhibiting the expression of PAX3 using antisense RNA in melanoma cell lines resulted in dose dependent reduction of proliferation of melanoma cells and induction of apoptosis, suggesting role for PAX3 in melanoma cell survival [78, 79]. Pax3 reduction also induces a loss of melanogenesis in melanoma cells, followed by a sharp decrease in MITF mRNA and 
gene promoter activity. Using microarray analyses PAX3 was identified as a regulator of the melanoma susceptibility and progression genes SCF, TGF- $\beta$, MUC18, RhoC, and TIMP3 [84-86], and this data has been represented in Figure 1 and Table 1.

PAX3 involved in muscle development is also implicated in tumorigenesis of muscle, rhabdomyosarcoma. Rhabdomyosarcoma is the most frequent soft tissue tumor in children under 15 years old. It develops as a consequence of disruption to the regulation of the growth and differentiation of myogenic precursor cells. PAX3 promotes tumorigenesis by influencing downstream MET pathway [87]. Rhabdomyosarcoma cells that undergo apoptosis due to specific antisense treatment is rescued from death by ectopic expression of PAX3, which indicates the prosurvival properties promoted by PAX3 [88]. PAX3 expression was markedly higher in gastric cancer tissues than in adjacent noncancerous tissues. MiR-206 significantly suppressed gastric cancer cell invasion and metastasis both in vitro and in vivo by downregulating PAX3. In vivo metastasis assay demonstrates that overexpression of PAX3 significantly promotes invasiveness and pulmonary metastasis of gastric cancer cells [35] (Table 1).

The above-mentioned studies clearly establish the oncogenic role of aberrant expression of PAX3 as a whole molecule in various cancers. Interestingly, $P A X 3$ gene is involved in gene translocations which results in fusion proteins involving PAX3 that offers them oncogenic potential [89]. PAX3FKHR (forkhead in rhabdomyosarcoma) fusion proteins are involved in promoting different molecular pathogeneses of alveolar rhabdomyosarcoma and embryonal rhabdomyosarcoma by inducing cellular transformation and preventing apoptosis $[89,90]$. The tumor promoting effects of PAX3 and PAX3-FKHR in rhabdomyosarcoma tumorigenesis are suggested to be through altering the MET, PTEN, or AP2 signaling pathways (Figure 1, Table 1). PAX3-FKHR mutants through gain of function promote tumor development in myoblasts, which again shows the oncogenic potential of PAX3 transcriptional activity [91]. Interestingly, PAX3-FKHR shows oncogenic effects, predominantly at relatively low levels, while at higher expression levels it induces suppression of growth in 3T3 L1 fibroblasts [92]. This particular study highlighted domain specific function of PAX3 at different levels of activity. At low levels, through its homeo domain and its associated function induced the transformation of 3T3L1 fibroblasts, while at higher levels they inhibited the transformation and growth potential of these cells, by its activity through paired domain. These studies clearly indicate a multifaceted dynamic function of PAX3 depending on the level of activity and cell type.

\section{Tumor Suppressing Role of PAX3}

The proliferation promoting characteristics of PAX3 have been well established in several cancers as discussed above. However, designating PAX3 as an oncogene has been impaired by contradicting reports available that highlight the growth inhibiting potential of PAX3. The ectopic expression of PAX3 dramatically inhibited thyroid cancer cell proliferation, colony formation, migration and invasion, induced cell cycle arrest, and apoptosis and retarded tumorigenic potential in nude mice [36]. These reports implicate PAX3 as a novel functional tumor suppressor in thyroid cancer, through inhibiting the activities of PI3K/Akt and MAPK signaling pathways and promoting transcription factor FOXO3a. The study also highlighted that epigenetic alteration such as promoter methylation as a major mechanism by which PAX3 is inactivated in this cancer (Figure 2 and Table 1). Further, PAX3 is a member that belongs to a group of transcription factors that are regulated by posttranslational modification such as acetylation. Pax3 acetylation decreased its transcriptional activity, thereby modulating the activity of downstream effectors Hes1 and increased Neurog2, resulting in sensory neuron differentiation [93]. Overexpression of miR-29 and 206 downregulates the expression of cell cycle genes and induces cell cycle arrest through stabilization of PAX3 in rhabdomyosarcoma, suggesting a tumor suppressor role for PAX3 [6].

\section{FOXO-PAX3 Association in Tumor Suppression}

PAX3, in addition to its tumor suppressor function, can also combine with other transcription factors and can regulate the expression of a wide variety of proteins that can act as tumor suppressors. FOXO1 transcription factors belong to the FOXO family and are involved in regulating key physiological pathways. In ARMS, gene translocations between $P A X 3$ and FOXO1 resulted in a fusion protein PAX3-FOXO1 which promoted the growth suppressive activity by upregulating the expression of gremlin 1 (GREM1) and death associated protein kinase-1 (DAPK1) tumor suppressor genes [96] (Figure 2). This study also characterized four downstream targets of PAX3-FOXO1 that contribute to the biological activities of growth suppression and myogenic differentiation [96]. PAX3-FOXO1 upregulated Noxa, which in turn promoted the activation of caspases and induced apoptosis. This particular signaling axis is considered as an important aspect of ARMS tumor biology that provides opportunity to create a therapeutic window by induction of apoptosis in ARMS cells [95] (Figure 1 and Table 1).

Similarly, PAX3 can modulate the expression of FOXO3, another type of transcription factor that belongs to the FOXO family that are involved in multiple signaling pathways and plays critical roles in a number of physiologic and pathologic processes [97]. Cancer patients with low FOXO3a expression had poor disease-free survival, cancer-specific survival, and overall survival [94].

FOXO3a negatively regulated Twistl and Y-box-binding protein 1 (YB-1) and positively regulated E-cadherin in KK47 and TCCsup cells that expressed Twist1, but not in T24 cells that did not express Twistl (Table 1). This suggests that FOXO3a could act as a prognostic factor in urothelial cancer and could represent a promising molecular target for cancer therapeutics [94]. Interestingly, FOXO3 partners with $\mathrm{PAX} 3 / 7$ to coordinately recruit RNA polymerase II 


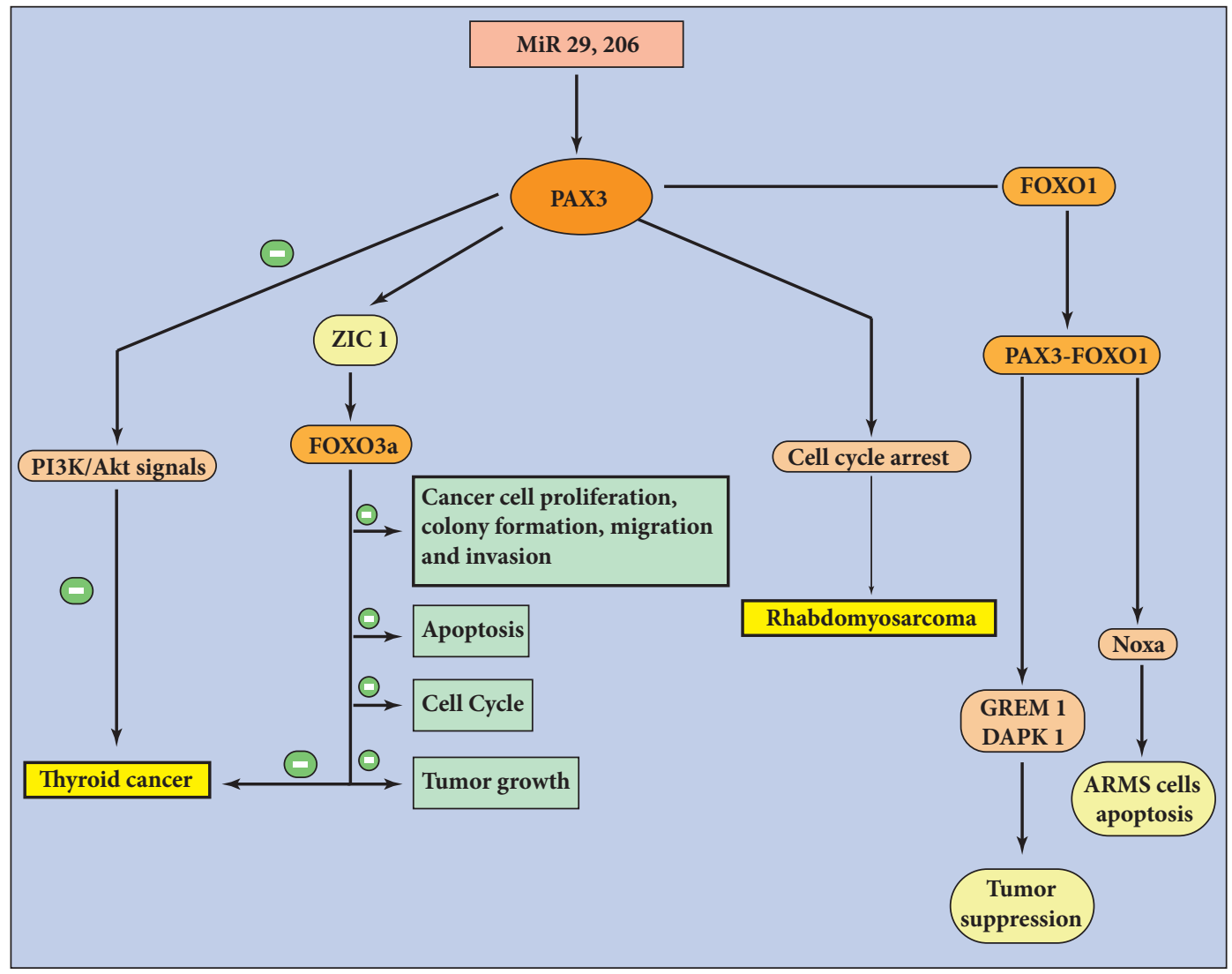

FIGURE 2: Tumor suppressor role of PAX3 in various types of cancer. Ectopic expression of PAX3 inhibits thyroid cancer cell proliferation, colony formation, migration, and invasion, and induced cell cycle arrest and leads to apoptosis through inhibiting the activities of PI3K/Akt and MAPK signaling pathways and promoting transcription factor FOXO3a. MiR 29 and 206 stabilize the PAX3 expression and induce the cell cycle arrest in the rhabdomyosarcoma cell lines. Fusion between the PAX3-FOXO1 induces the tumor cell suppression by inducing the expression of GERM1 and DAPK1 genes which in turn upregulate NOXA (promote activation of caspases and apoptosis) and induce apoptosis in ARMS cells.

and form a pre initiation complex (PIC) to activate MyoD transcription in myoblasts leading to its differentiation [98]. The ectopic expression of PAX3, upregulated the expression of ZIC1, a zinc-finger transcription factor, which in turn increases FOXO3a transcriptional activity in thyroid cancer cells. The regulation of ZIC1 by PAX3 suggests that PAX3 regulates the expression and activity of FOXO3a through multiple mechanisms in thyroid cancer [99]. These studies clearly indicate that, in addition to its own tumor suppressor role, PAX3 can promote tumor suppressor function by interplaying FOXO family of transcription factors to promote the expression of tumor suppressor genes that are controlled by them.

\section{Conclusion}

PAX3 is a pivotal gene involved in organ development and is also known to play a very important role in development of cancer. PAX3 regulates cell differentiation, proliferation, migration, and the survival of different cell types through activating several target genes. Majority of studies on PAX3 clearly indicate a crucial role for it in the oncogenesis of several human tumors. Interestingly, the studies involving ectopic expression of PAX3 in thyroid cancers show that it can act as a tumor suppressor. Further, PAX3 in association with FOXO3a transcription factor modulates several pathways that lead to cellular differentiation and growth inhibition in cancer. These facts clearly prevent us from designating PAX3 as an oncogene, as there are results pointing in other direction (Figure 3). The possibility of PAX3 having dual functions as oncogenic and tumor suppressor based on the cell type, context, and physiologic stimuli cannot be ignored. While the oncogenic role has been well established, more studies are required that can strengthen its tumor suppressor role. Studies reporting their antiproliferative effect should not be ignored and should be considered seriously. Due to the existing discrepancies, it is important to understand the exact role of PAX3 in diseases, where PAX3 expression is altered. Future studies should focus on determining the tissues specific functions of PAX3, where it is either mutated or aberrantly expressed. This vital information is important and will be very useful in developing therapeutic strategies targeting PAX3. 


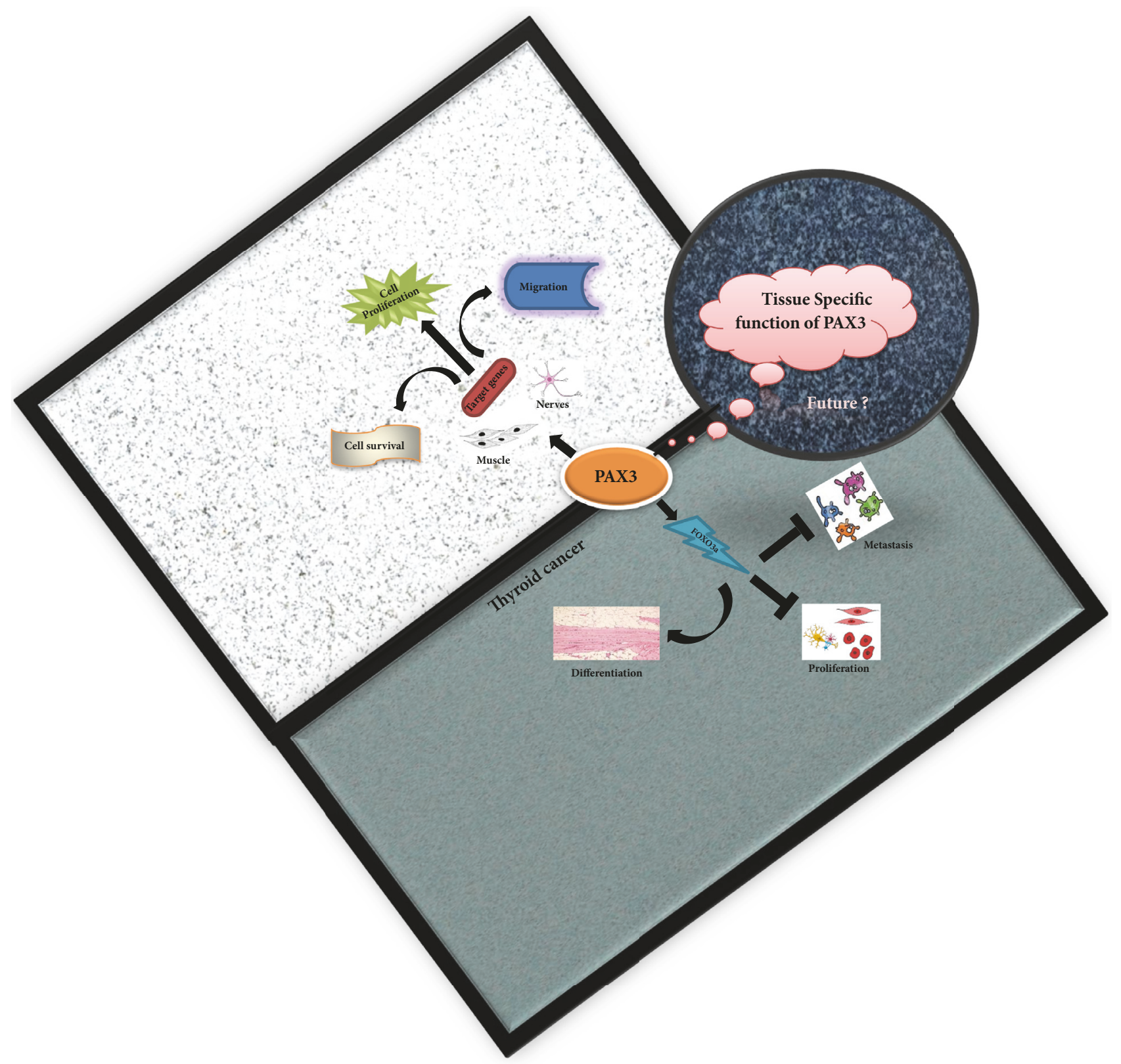

FIGURE 3: Schematic model explaining various tissue-specific cellular functions of PAX3 in normal and disease conditions. PAX3 regulates cell differentiation, proliferation, migration, and the survival of different cell types through activating several target genes. Ectopic expression of PAX3 in thyroid cancers shows that it can act as a tumor suppressor. PAX3 in association with FOXO3a leads to cellular differentiation and growth inhibition in cancer. Determining tissue-specific effects of PAX3 is necessary to understand its role in disease pathogenesis.

\section{Abbreviations}

PAX3:

Paired box gene3

FKHR (FOXOla): Forkhead transcription factor

MTIF: Microphthalmia-associated transcription factor

SCF:

TGF- $\beta$ :

MUC 18:

RhoC:

TIMP3:

\section{Stem cell factor}

Transforming growth factor beta 1 Melanoma cell adhesion molecule Ras Homolog Family Member C Tissue inhibitor of metalloproteinase 3
YB-1: $\quad$ Y-box Binding Protein-1

FOXO3a: Forkhead box O3

PI3K: Phosphoinositide 3-kinase

Akt: $\quad$ Protein Kinase B

MAPK: Mitogen-activated protein kinase

ZIC1: $\quad$ Zinc-finger transcription factor.

\section{Conflicts of Interest}

The authors declarethat they have no conflicts of interest. 


\section{Acknowledgments}

University Grants Commission-Basic Science Research (UGC-BSR) student fellowship in sciences for supporting Mr. Ashok Arasu is highly acknowledged. Department of Biotechnology (DBT), Ramalingaswami Reentry Fellowship Program for supporting Dr. Thirunavukkarasu Velusamy is gratefully acknowledged.

\section{References}

[1] L. V. Nguyen, R. Vanner, P. Dirks, and C. J. Eaves, "Cancer stem cells: an evolving concept," Nature Reviews Cancer, vol. 12, no. 2, pp. 133-143, 2012.

[2] C. G. Li and M. R. Eccles, "PAX genes in cancer; friends or foes?" Frontiers in Genetics, vol. 3, 2012.

[3] Q. Wang, W.-H. Fang, J. Krupinski, S. Kumar, M. Slevin, and P. Kumar, "Pax genes in embryogenesis and oncogenesis: Genes...," Journal of Cellular and Molecular Medicine, vol. 12, no. 6A, pp. 2281-2294, 2008.

[4] A. Buchberger, D. Freitag, and H.-H. Arnold, "A homeo-paired domain-binding motif directs Myf5 expression in progenitor cell of limb muscle," Development, vol. 134, no. 6, pp. 1171-1180, 2007.

[5] E. J. D. Robson, S. He, and M. R. Eccles, "A PANorama of PAX genes in cancer and development," Nature Reviews Cancer, vol. 6, no. 1, pp. 52-62, 2006.

[6] L. Li, A. L. Sarver, S. Alamgir, and S. Subramanian, "Downregulation of microRNAs miR-1, -206 and -29 stabilizes PAX3 and CCND2 expression in rhabdomyosarcoma," Laboratory Investigation, vol. 92, no. 4, pp. 571-583, 2012.

[7] F. G. Barr, N. Galili, J. Holick, J. A. Biegel, G. Rovera, and B. S. Emanuel, "Rearrangement of the PAX3 paired box gene in the paediatric solid tumour alveolar rhabdomyosarcoma," Nature Genetics, vol. 3, no. 2, pp. 113-117, 1993.

[8] R. J. Davis, C. M. D’Cruz, M. A. Lovell, J. A. Biegel, and F. G. Barr, "Fusion of PAX7 to FKHR by the variant $\mathrm{t}(1 ; 13)(\mathrm{p} 36 ; \mathrm{q} 14)$ translocation in alveolar rhabdomyosarcoma," Cancer Research, vol. 54, no. 11, pp. 2869-2872, 1994.

[9] A. M. Morrison, S. L. Nutt, C. Thévenin, A. Rolink, and M. Busslinger, "Loss- and gain-of-function mutations reveal an important role of BSAP (Pax-5) at the start and end of B cell differentiation," Seminars in Immunology, vol. 10, no. 2, pp. 133$142,1998$.

[10] T. G. Kroll, P. Sarraf, L. Pecciarini et al., "PAX8-PPAR $\gamma 1$ fusion in oncogene human thyroid carcinoma," Science, vol. 289, no. 5483, pp. 1357-1360, 2000.

[11] G. Cazzaniga, M. Daniotti, S. Tosi et al., "The paired box domain gene PAX5 is fused to ETV6/TEL in an acute lymphoblastic leukemia case," Cancer Research, vol. 61, no. 12, pp. 4666-4670, 2001.

[12] A. R. Marques, C. Espadinha, A. L. Catarino et al., "Expression of PAX8-PPAR $\gamma 1$ rearrangements in both follicular thyroid carcinomas and adenomas," The Journal of Clinical Endocrinology \& Metabolism, vol. 87, no. 8, pp. 3947-3952, 2002.

[13] R. G. Harris, E. White, E. S. Phillips, and K. A. Lillycrop, "The expression of the developmentally regulated proto-oncogene Pax-3 is modulated by N-Myc," The Journal of Biological Chemistry, vol. 277, no. 38, pp. 34815-34825, 2002.

[14] S. Du, E. J. Lawrence, D. Strzelecki et al., "Co-expression of alternatively spliced forms of PAX3, PAX7, PAX3-FKHR and
PAX7-FKHR with distinct DNA binding and transactivation properties in rhabdomyosarcoma," International Journal of Cancer, vol. 115, no. 1, pp. 85-92, 2005.

[15] J. D. Kubic, K. P. Young, R. S. Plummer, A. E. Ludvik, and D. Lang, "Pigmentation PAX-ways: The role of Pax3 in melanogenesis, melanocyte stem cell maintenance, and disease," Pigment Cell \& Melanoma Research, vol. 21, no. 6, pp. 627-645, 2008.

[16] K. Tsukamoto, Y. Nakamura, and N. Niikawa, "Isolation of two isoforms of the PAX3 gene transcripts and their tissue-specific alternative expression in human adult tissues," Human Genetics, vol. 93, no. 3, pp. 270-274, 1994.

[17] T. D. Barber, M. C. Barber, T. E. Cloutier, and T. B. Friedman, "PAX3 gene structure, alternative splicing and evolution," Gene, vol. 237, no. 2, pp. 311-319, 1999.

[18] C. J. Parker, S. G. Shawcross, H. Li et al., "Expression of PAX 3 alternatively spliced transcripts and identification of two new isoforms in human tumors of neural crest origin," International Journal of Cancer, vol. 108, no. 2, pp. 314-320, 2004.

[19] Q. Wang, S. Kumar, M. Slevin, and P. Kumar, "Functional analysis of alternative isoforms of the transcription factor PAX3 in melanocytes in vitro," Cancer Research, vol. 66, no. 17, pp. 8574-8580, 2006.

[20] O. Sanchez-Ferras, B. Coutaud, T. D. Samani, I. Tremblay, O. Souchkova, and N. Pilon, "Caudal-related homeobox (Cdx) protein-dependent integration of canonical Wnt signaling on Paired-box 3 (Pax3) neural crest enhancer," The Journal of Biological Chemistry, vol. 287, no. 20, pp. 16623-16635, 2012.

[21] M. D. Goulding, G. Chalepakis, U. Deutsch, J. R. Erselius, and P. Gruss, "Pax-3, a novel murine DNA binding protein expressed during early neurogenesis," EMBO Journal, vol. 10, no. 5, pp. 1135-1147, 1991.

[22] D. Lang, M. M. Lu, L. Huang et al., "Pax3 functions at a nodal point in melanocyte stem cell differentiation," Nature, vol. 433, no. 7028, pp. 884-887, 2005.

[23] F. Relaix, D. Rocancourt, A. Mansouri, and M. Buckingham, "A $\mathrm{Pax} 3 / \mathrm{Pax} 7$-dependent population of skeletal muscle progenitor cells," Nature, vol. 435, no. 7044, pp. 948-953, 2005.

[24] A.-H. Monsoro-Burq, E. Wang, and R. Harland, "Msx1 and Pax3 cooperate to mediate FGF8 and WNT signals during Xenopus neural crest induction," Developmental Cell, vol. 8, no. 2, pp. 167-178, 2005.

[25] S. J. Conway, D. J. Henderson, and A. J. Copp, "Pax3 is required for cardiac neural crest migration in the mouse: Evidence from the splotch $(\mathrm{Sp}(2 \mathrm{H}))$ mutant," Development, vol. 124, no. 2, pp. 505-514, 1997.

[26] M. Olaopa, H.-M. Zhou, P. Snider et al., "Pax3 is essential for normal cardiac neural crest morphogenesis but is not required during migration nor outflow tract septation," Developmental Biology, vol. 356, no. 2, pp. 308-322, 2011.

[27] D. J. Epstein, M. Vekemans, and P. Gros, "splotch (Sp2H), a mutation affecting development of the mouse neural tube, shows a deletion within the paired homeodomain of Pax-3," Cell, vol. 67, no. 4, pp. 767-774, 1991.

[28] L. Pani, M. Horal, and M. R. Loeken, "Rescue of neural tube defects in Pax-3-deficient embryos by p53 loss of function: Implications for Pax-3-dependent development and tumorigenesis," Genes \& Development, vol. 16, no. 6, pp. 676-680, 2002.

[29] R. A. Macina, F. G. Barr, N. Galili, and H. C. Riethman, "Genomic organization of the human PAX3 gene: DNA sequence analysis of the region disrupted in alveolar rhabdomyosarcoma," Genomics, vol. 26, no. 1, pp. 1-8, 1995. 
[30] L. Xia, Q. Huang, D. Nie et al., "PAX3 is overexpressed in human glioblastomas and critically regulates the tumorigenicity of glioma cells," Brain Research, vol. 1521, pp. 68-78, 2013.

[31] W.-H. Fang, Q. Wang, H.-M. Li, M. Ahmed, P. Kumar, and S. Kumar, "PAX3 in neuroblastoma: Oncogenic potential, chemosensitivity and signalling pathways," Journal of Cellular and Molecular Medicine, vol. 18, no. 1, pp. 38-48, 2014.

[32] R. S. Plummer, C. R. Shea, M. Nelson et al., "PAX3 expression in primary melanomas and nevi," Modern Pathology, vol. 21, no. 5, pp. 525-530, 2008.

[33] E. Frascella, L. Toffolatti, and A. Rosolen, "Normal and rearranged PAX3 expression in human rhabdomyosarcoma," Cancer Genetics and Cytogenetics, vol. 102, no. 2, pp. 104-109, 1998.

[34] T. W. Schulte, J. A. Toretsky, E. Ress, L. Helman, and L. M. Neckers, "Expression of PAX3 in Ewing's sarcoma family of tumors," Biochemical and Molecular Medicine, vol. 60, no. 2, pp. 121-126, 1997.

[35] L. Zhang, L. Xia, L. Zhao et al., "Activation of PAX3-MET pathways due to miR-206 loss promotes gastric cancer metastasis," Carcinogenesis, vol. 36, no. 3, pp. 390-399, 2014.

[36] W. Liu, F. Sui, J. Liu et al., "PAX3 is a novel tumor suppressor by regulating the activities of major signaling pathways and transcription factor FOXO3a in thyroid cancer," Oncotarget, vol. 7, no. 34, pp. 54744-54757, 2016.

[37] C. M. Linardic, "PAX3-FOXO1 fusion gene in rhabdomyosarcoma," Cancer Letters, vol. 270, no. 1, pp. 10-18, 2008.

[38] B. L. Nelms and P. A. Labosky, "Transcriptional Control of Neural Crest Development," Colloquium Series on Developmental Biology, vol. 1, no. 1, pp. 1-227, 2010.

[39] M. Buckingham and F. Relaix, "PAX3 and PAX7 as upstream regulators of myogenesis," Seminars in Cell \& Developmental Biology, vol. 44, pp. 115-125, 2015.

[40] G. N. Serbedzija and A. P. McMahon, "Analysis of neural crest cell migration in splotch mice using a neural Crest-Specific LacZ reporter," Developmental Biology, vol. 185, no. 2, pp. 139147, 1997.

[41] M. Zhao, S. C. Isom, H. Lin et al., "Tracing the stemness of porcine skin-derived progenitors (pSKP) back to specific marker gene expression," Cloning and Stem Cells, vol. 11, no. 1, pp. 111-122, 2009.

[42] M. Wu, J. Li, K. A. Engleka et al., "Persistent expression of Pax3 in the neural crest causes cleft palate and defective osteogenesis in mice," The Journal of Clinical Investigation, vol. 118, no. 6, pp. 2076-2087, 2008.

[43] D. J. Henderson, P. Ybot-Gonzalez, and A. J. Copp, "Overexpression of the chondroitin sulphate proteoglycan versican is associated with defective neural crest migration in the Pax3 mutant mouse (splotch)," Mechanisms of Development, vol. 69, no. 1-2, pp. 39-51, 1997.

[44] C.-S. Hong and J.-P. Saint-Jeannet, "The activity of Pax3 and Zicl regulates three distinct cell fates at the neural plate border," Molecular Biology of the Cell (MBoC), vol. 18, no. 6, pp. 21922202, 2007.

[45] T. Sato, N. Sasai, and Y. Sasai, "Neural crest determination by co-activation of Pax3 and Zicl genes in Xenopus ectoderm," Development, vol. 132, no. 10, pp. 2355-2363, 2005.

[46] T. Burstyn-Cohen, J. Stanleigh, D. Sela-Donenfeld, and C. Kalcheim, "Canonical Wnt activity regulates trunk neural crest delamination linking BMP/noggin signaling with G1/S transition," Development, vol. 131, no. 21, pp. 5327-5339, 2004.
[47] L. A. Taneyhill and M. Bronner-Fraser, "Dynamic alterations in gene expression, after Wnt-mediated induction of avian neural crest," Molecular Biology of the Cell (MBoC), vol. 16, no. 11, pp. 5283-5293, 2005.

[48] H. Nakazaki, A. C. Reddy, B. L. Mania-Farnell et al., "Key basic helix-loop-helix transcription factor genes Hes1 and Ngn2 are regulated by Pax3 during mouse embryonic development," Developmental Biology, vol. 316, no. 2, pp. 510-523, 2008.

[49] S. A. Koblar, M. Murphy, G. L. Barrett, A. Underhill, P. Gros, and P. F. Bartlett, "Pax-3 regulates neurogenesis in neural crestderived precursor cells," Journal of Neuroscience Research, vol. 56, no. 5, pp. 518-530, 1999.

[50] S. J. Conway, R. E. Godt, C. J. Hatcher et al., "Neural crest is involved in development of abnormal myocardial function," Journal of Molecular and Cellular Cardiology, vol. 29, no. 10, pp. 2675-2685, 1997.

[51] S. J. Conway, D. J. Henderson, M. L. Kirby, R. H. Anderson, and A. J. Copp, "Development of a lethal congenital heart defect in the splotch (Pax3) mutant mouse," Cardiovascular Research, vol. 36, no. 2, pp. 163-173, 1997.

[52] L. J. Manderfield, K. A. Engleka, H. Aghajanian et al., "Pax3 and hippo signaling coordinate melanocyte gene expression in neural crest," Cell Reports, vol. 9, no. 5, pp. 1885-1895, 2014.

[53] Y. J. Choi, T. J. Yoon, and Y. H. Lee, "Changing expression of the genes related to human hair graying," European Journal of Dermatology, vol. 18, no. 4, pp. 397-399, 2008.

[54] T. M. Lamey, A. Koenders, and M. Ziman, "Pax genes in myogenesis: Alternate transcripts add complexity," Histology and Histopathology, vol. 19, no. 4, pp. 1289-1300, 2004.

[55] J. A. Epstein, J. Li, D. Lang et al., "Migration of cardiac neural crest cells in Splotch embryos," Development, vol. 127, no. 9, pp. 1869-1878, 2000.

[56] P. Bailey, T. Holowacz, and A. B. Lassar, "The origin of skeletal muscle stem cells in the embryo and the adult," Current Opinion in Cell Biology, vol. 13, no. 6, pp. 679-689, 2001.

[57] S. J. Odelberg, A. Kollhoff, and M. T. Keating, "Dedifferentiation of mammalian myotubes induced by msxl," Cell, vol. 103, no. 7, pp. 1099-1109, 2000.

[58] K. A. Miller, J. Barrow, J. M. Collinson et al., "A highly conserved Wnt-dependent TCF4 binding site within the proximal enhancer of the anti-myogenic Msxl gene supports expression within Pax3-expressing limb bud muscle precursor cells," Developmental Biology, vol. 311, no. 2, pp. 665-678, 2007.

[59] A. Karaman and C. Aliagaoglu, "Waardenburg syndrome type 1," Dermatology online journal, vol. 12, no. 3, 2003, https://escholarship.org/uc/item/0r5371ck.

[60] M. Ptok and S. Morlot, "Unilateral sensineural deafness associated with mutations in the PAX3-gene in Waardenburg syndrome type I," HNO, vol. 54, no. 7, pp. 557-560, 2006.

[61] A. P. Read and V. E. Newton, "Waardenburg syndrome," Journal of Medical Genetics, vol. 34, no. 8, pp. 656-665, 1997.

[62] M. Tassabehji, A. P. Read, V. E. Newton et al., "Mutations in the PAX3 gene causing Waardenburg syndrome type 1 and type 2," Nature Genetics, vol. 3, no. 1, pp. 26-30, 1993.

[63] M. Tassabehjl, V. E. Newton, K. Leverton et al., "PAX3 gene structure and mutations: Close analogies between waardenburg syndrome and the Splotch mouse," Human Molecular Genetics, vol. 3, no. 7, pp. 1069-1074, 1994.

[64] M. Tassabehji, V. E. Newton, X.-Z. Liu et al., "The mutational spectrum in waardenburg syndrome," Human Molecular Genetics, vol. 4, no. 11, pp. 2131-2137, 1995. 
[65] G. Van Camp, M. N. Van Thienen, I. Handig et al., "Chromosome 13q deletion with Waardenburg syndrome: Further evidence for a gene involved in neural crest function on 13q," Journal of Medical Genetics, vol. 32, no. 7, pp. 531-536, 1995.

[66] A. Muratovska, C. Zhou, S. He, P. Goodyer, and M. R. Eccles, "Paired-Box genes are frequently expressed in cancer and often required for cancer cell survival," Oncogene, vol. 22, no. 39, pp. 7989-7997, 2003.

[67] T. Glaser, D. S. Walton, and R. L. Maas, "Genomic structure, evolutionary conservation and aniridia mutations in the human PAX6 gene," Nature Genetics, vol. 2, no. 3, pp. 232-239, 1992.

[68] T. Jordan, I. Hanson, D. Zaletayev et al., "The human PAX6 gene is mutated in two patients with aniridia," Nature Genetics, vol. 1, no. 5, pp. 328-332, 1992.

[69] M. Tassabehji, A. P. Read, V. E. Newton et al., "Waardenburg's syndrome patients have mutations in the human homologue of the Pax-3 paired box gene," Nature, vol. 355, no. 6361, pp. 635636, 1992.

[70] P. Sanyanusin, L. A. Schimmenti, L. A. McNoe et al., "Mutation of the PAX2 gene in a family with optic nerve colobomas, renal anomalies and vesicoureteral reflux," Nature Genetics, vol. 9, no. 4, pp. 358-364, 1995.

[71] M. Bouchard, A. Souabni, M. Mandler, A. Neubüser, and M. Busslinger, "Nephric lineage specification by Pax2 and Pax8," Genes \& Development, vol. 16, no. 22, pp. 2958-2970, 2002.

[72] F. Francioso, F. Carinci, L. Tosi et al., "Identification of Differentially Expressed Genes in Human Salivary Gland Tumors by DNA Microarrays 1 Supported by Università di Ferrara, Murst Prin, Carisbo, Carife Grants," Molecular Cancer Therapeutics, vol. 1, no. 7, pp. 533-538, 2002.

[73] N. Chi and J. A. Epstein, "Getting your Pax straight: pax proteins in development and disease," Trends in Genetics, vol. 18, no. 1, pp. 41-47, 2002.

[74] D. Lang, S. K. Powell, R. S. Plummer, K. P. Young, and B. A. Ruggeri, "PAX genes: roles in development, pathophysiology, and cancer," Biochemical Pharmacology, vol. 73, no. 1, pp. 1-14, 2007.

[75] C. C. Maulbecker and P. Gruss, "The oncogenic potential of Pax genes," EMBO Journal, vol. 12, no. 6, pp. 2361-2367, 1993.

[76] M. Jothi, K. Nishijo, C. Keller, and A. K. Mal, "AKT and PAX3FKHR cooperation enforces myogenic differentiation blockade in alveolar rhabdomyosarcoma cell," Cell Cycle, vol. 11, no. 5, pp. 895-908, 2012.

[77] T. R. Gershon, O. Oppenheimer, S. S. Chin, and W. L. Gerald, "Temporally regulated neural crest transcription factors distinguish neuroectodermal tumors of varying malignancy and differentiation," Neoplasia, vol. 7, no. 6, pp. 575-584, 2005.

[78] F. A. Scholl, J. Kamarashev, O. V. Murmann, R. Geertsen, R. Dummer, and B. W. Schäfer, "PAX3 is expressed in human melanomas and contributes to tumor cell survival," Cancer Research, vol. 61, no. 3, pp. 823-826, 2001.

[79] S.-J. He, G. Stevens, A. W. Braithwaite, and M. R. Eccles, "Transfection of melanoma cells with antisense PAX3 oligonucleotides additively complements cisplatin-induced cytotoxicity," Molecular Cancer Therapeutics, vol. 4, no. 6, pp. 996-1003, 2005.

[80] X. D. Wang, S. C. Morgan, and M. R. Loeken, "Pax3 stimulates p53 ubiquitination and degradation independent of transcription," PLoS ONE, vol. 6, no. 12, Article ID e29379, 2011.

[81] Q. Wang, C. Parker, S. Kumar, and P. Kumar, "Differential PAX3 expression in neuroblastoma," in Proceedings of the Differential PAX3 expression in neuroblastoma, vol. 3, p. 271, 2004.
[82] K. W. Vance and C. R. Goding, "The transcription network regulating melanocyte development and melanoma," Pigment Cell Research, vol. 17, no. 4, pp. 318-325, 2004.

[83] J. A. Blake and M. R. Ziman, "Pax3 transcripts in melanoblast development," Development, Growth \& Differentiation, vol. 47, no. 9, pp. 627-635, 2005.

[84] A. K. Kamaraju, C. Bertolotto, J. Chebath, and M. Revel, "Pax3 down-regulation and shut-off of melanogenesis in melanoma B16/F10.9 by interleukin-6 receptor signaling," The Journal of Biological Chemistry, vol. 277, no. 17, pp. 15132-15141, 2002.

[85] C. S. K. Mayanil, D. George, L. Freilich et al., "Microarray Analysis Detects Novel Pax3 Downstream Target Genes," The Journal of Biological Chemistry, vol. 276, no. 52, pp. 4929949309, 2001.

[86] Q. Wang, S. Kumar, N. Mitsios, M. Slevin, and P. Kumar, "Investigation of downstream target genes of PAX3c, PAX3e and PAX3g isoforms in melanocytes by microarray analysis," International Journal of Cancer, vol. 120, no. 6, pp. 1223-1231, 2007.

[87] J. P. Ginsberg, R. J. Davis, J. L. Bennicelli, L. E. Nauta, and F. G. Barr, "Up-regulation of MET but not neural cell adhesion molecule expression by the PAX3-FKHR fusion protein in alveolar rhabdomyosarcoma," Cancer Research, vol. 58, no. 16, pp. 3542-3546, 1998.

[88] C. M. Margue, M. Bernasconi, F. G. Barr, and B. W. Schäfer, "Transcriptional modulation of the anti-apoptotic protein BCL-XL by the paired box transcription factors PAX3 and PAX3/FKHR," Oncogene, vol. 19, no. 25, pp. 2921-2929, 2000.

[89] P. Y. P. Lam, J. E. Sublett, A. D. Hollenbach, and M. F. Roussel, "The oncogenic potential of the Pax3-FKHR fusion protein requires the Pax3 homeodomain recognition helix but not the Pax3 paired-box DNA binding domain," Molecular and Cellular Biology, vol. 19, no. 1, pp. 594-601, 1999.

[90] M. Bernasconi, A. Remppis, W. J. Fredericks, F. J. Rauscher III, and B. W. Schäfer, "Induction of apoptosis in rhabdomyosarcoma cells through down-regulation of PAX proteins," Proceedings of the National Acadamy of Sciences of the United States of America, vol. 93, no. 23, pp. 13164-13169, 1996.

[91] Y. Zhang, J. Schwartz, and C. Wang, "Comparative analysis of paired- and homeodomain-specific roles in PAX3-FKHR oncogenesis," International Journal of Clinical and Experimental Pathology, vol. 2, no. 4, pp. 370-383, 2009.

[92] S. J. Xia and F. G. Barr, "Analysis of the transforming and growth suppressive activities of the PAX3-FKHR oncoprotein," Oncogene, vol. 23, no. 41, pp. 6864-6871, 2004.

[93] S. Ichi, V. Boshnjaku, Y.-W. Shen et al., "Role of Pax3 acetylation in the regulation of Hes1 and Neurog2," Molecular Biology of the Cell (MBoC), vol. 22, no. 4, pp. 503-512, 2011.

[94] M. Shiota, Y. Song, A. Yokomizo et al., "Foxo3a suppression of urothelial cancer invasiveness through twistl, Y-box-binding protein 1, and E-cadherin regulation," Clinical Cancer Research, vol. 16, no. 23, pp. 5654-5663, 2010.

[95] A. D. Marshall, F. Picchione, R. I. K. Geltink, and G. C. Grosveld, "PAX3-FOXO1 induces up-regulation of Noxa sensitizing alveolar rhabdomyosarcoma cells to apoptosis," Neoplasia, vol. 15, no. 7, pp. 738-748, 2013.

[96] E. H. Ahn, "Regulation of target genes of PAX3-FOXO1 in alveolar rhabdomyosarcoma," Anticancer Reseach, vol. 33, no. 5, pp. 2029-2035, 2013.

[97] Z. Fu and D. J. Tindall, "FOXOs, cancer and regulation of apoptosis," Oncogene, vol. 27, no. 16, pp. 2312-2319, 2008. 
[98] P. Hu, K. G. Geles, J.-H. Paik, R. A. DePinho, and R. Tjian, "Codependent Activators Direct Myoblast-Specific MyoD Transcription," Developmental Cell, vol. 15, no. 4, pp. 534-546, 2008.

[99] W. Qiang, Y. Zhao, Q. Yang et al., "ZIC1 is a putative tumor suppressor in thyroid cancer by modulating major signaling pathways and transcription factor FOXO3a," The Journal of Clinical Endocrinology \& Metabolism, vol. 99, no. 7, pp. E1163E1172, 2014. 


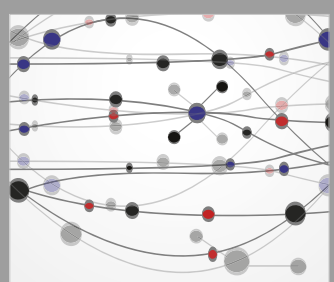

The Scientific World Journal
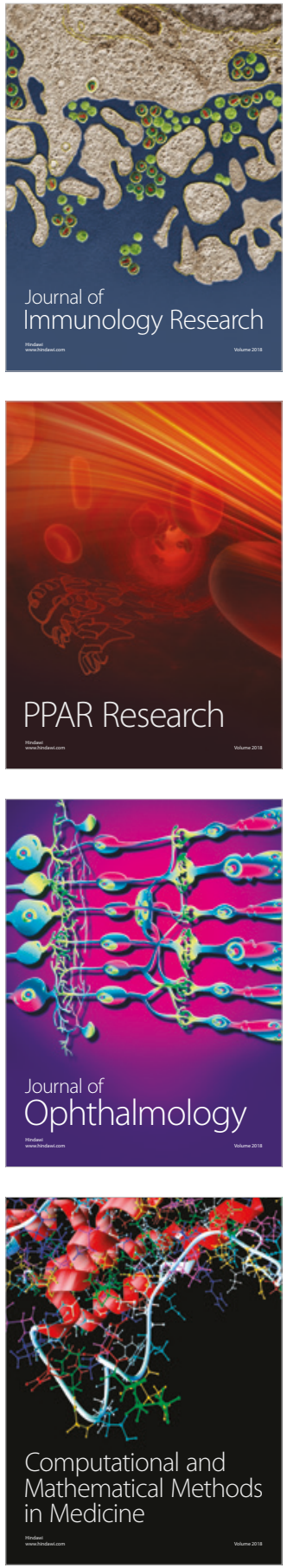

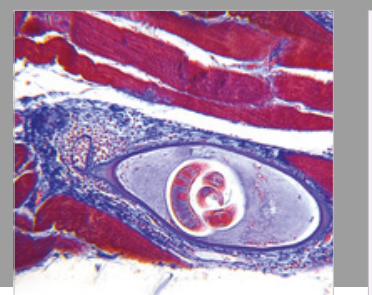

Gastroenterology Research and Practice

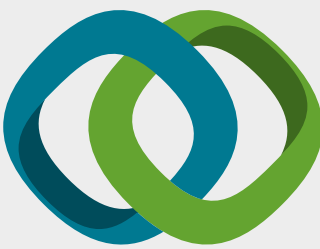

\section{Hindawi}

Submit your manuscripts at

www.hindawi.com
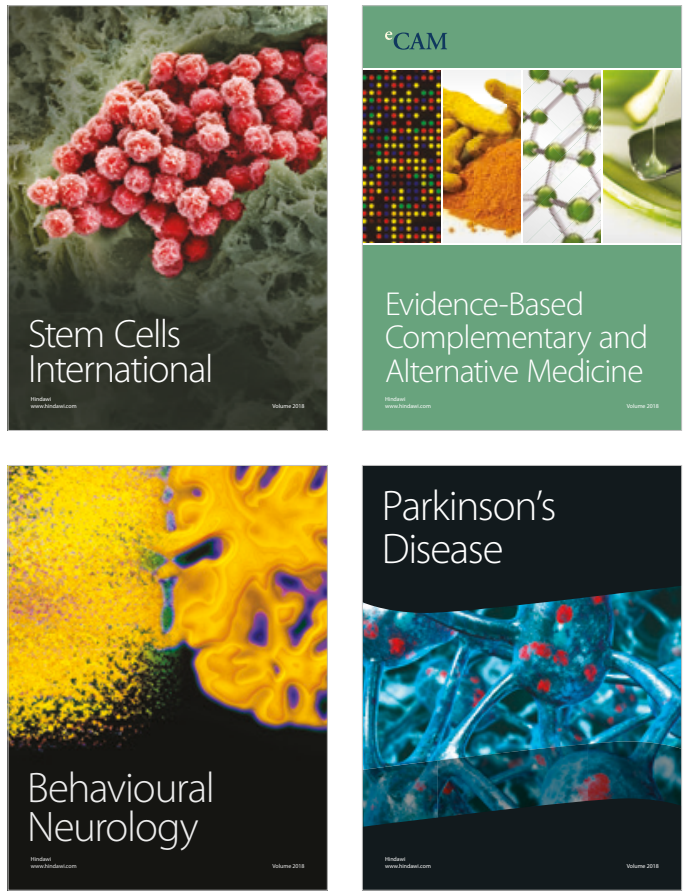

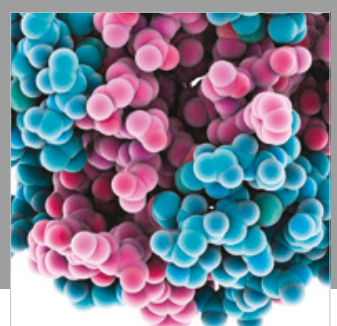

ournal of

Diabetes Research

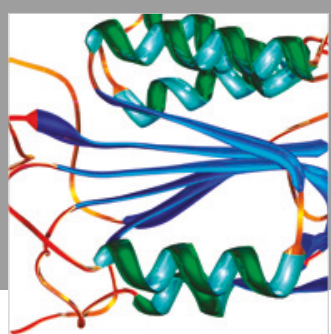

Disease Markers
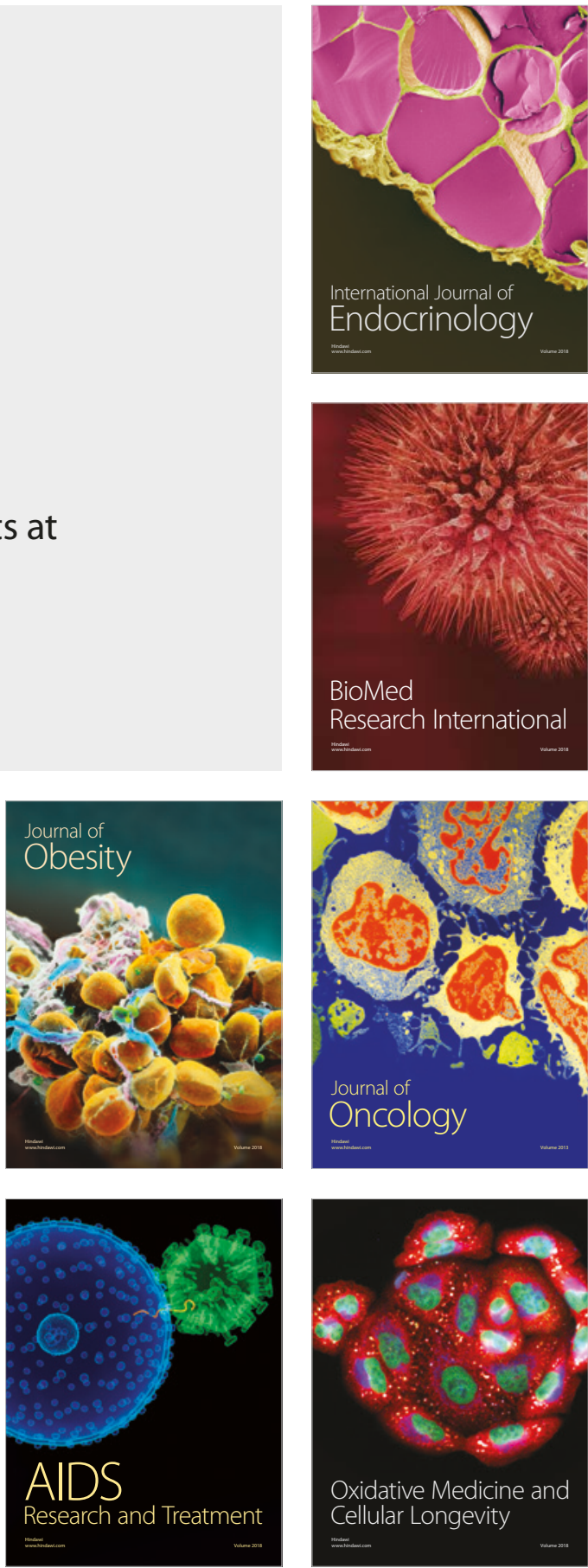